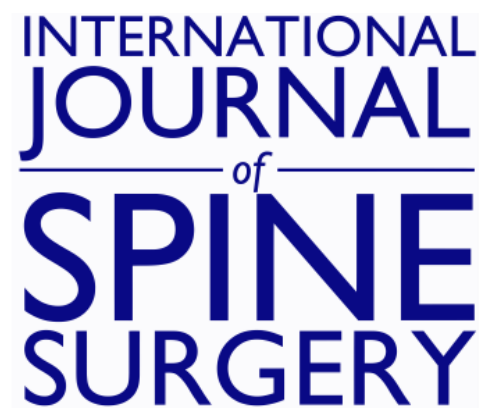

\title{
"Outside-in" Technique, Clinical Results, and Indications with Transforaminal Lumbar Endoscopic Surgery: a Retrospective Study on 220 Patients on Applied Radiographic Classification of Foraminal Spinal Stenosis
}

Kai-Uwe Lewandrowski

Int J Spine Surg 2014, 8 ()

doi: https://doi.org/10.14444/1026

http://ijssurgery.com/content/8/26

This information is current as of April 26, 2023.

Email Alerts Receive free email-alerts when new articles cite this article. Sign up at:

http://ijssurgery.com/alerts 


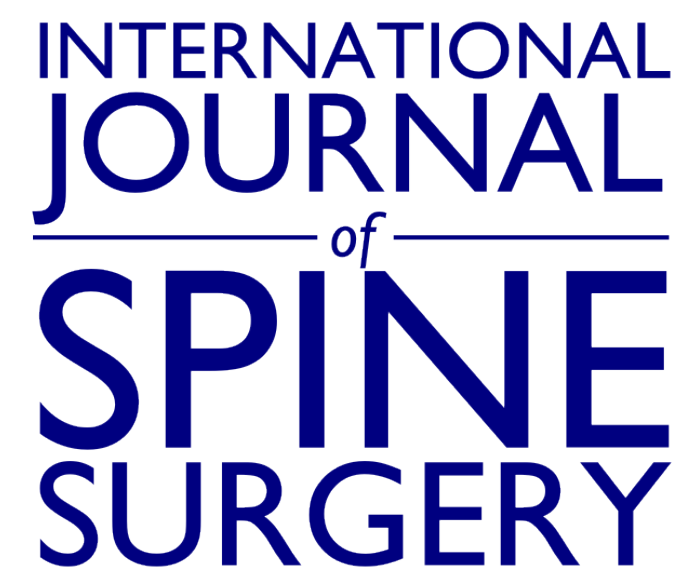

This article generously published free of charge by the International Society for the Advancement of Spine Surgery.

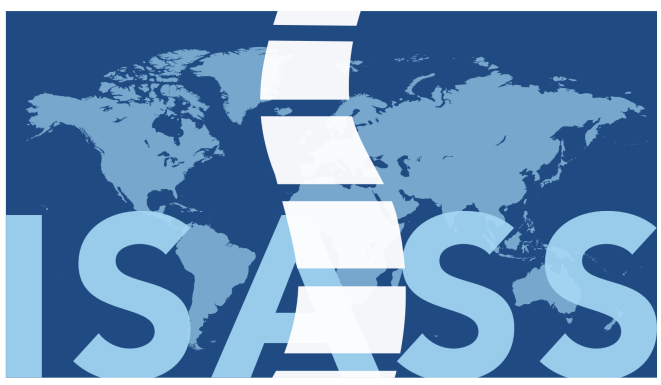

INTERNATIONAL SOCIETY for the ADVANCEMENT of SPINE SURGERY 


\section{"Outside-in" Technique, Clinical Results, and Indications with Transforaminal Lumbar Endoscopic Surgery: a Retrospective Study on 220 Patients on Applied Radiographic Classification of Foraminal Spinal Stenosis}

Kai-Uwe Lewandrowski, MD

Center for Advanced Spinal Surgery of Southern Arizona, Tucson, AZ

\section{Abstract}

\section{Objective}

To analyze and describe appropriate surgical indications for endoscopically performed transforaminal decompression with the outside-in technique with foraminoplasty in patients with lateral stenosis with and without herniated disc.

\section{Background and Significance}

Endoscopic microdiscectomy is growing in popularity for the removal of lumbar disc herniations. Recent advances in surgical techniques allow for percutaneous endoscopically assisted bony decompression as well.

\section{Materials and Methods}

A retrospective study of 220 consecutive patients undergoing percutaneous endoscopic transforaminal foraminoplasty and microdiscectomy at 228 levels was conducted with intent of identifying appropriate surgical indications in patients with monoradiculopathy. The mean follow up was 46 months ranging from 26 to 54 months. Preoperatively, foraminal and lateral recess stenosis was graded on preoperative MRI and CT scans by dividing the lumbar neuroforamen into three zones: a) entry zone, b) middle zone, and c) exit zone. In addition, the presence of disc herniation causing neural element compression in the lateral recess and neuroforamen was noted. Disc herniations, if present, were 
recorded as either extruded and contained disc herniations. Surgical outcomes were classified according to the Macnab criteria. In addition, reductions in VAS scores were assessed.

\section{Results}

According to the Macnab criteria, excellent and good results were obtained in $85 \%$ (186/ 220) of patients with monoradiculopathy. The mean VAS score decreased from $7.5 \pm 1.5$ preoperatively to $2.8 \pm 1.9$ at the final follow-up $(P<0.01)$. Concomitant extruded disc herniations and contained disc bulges were recorded in 24 and 82 patients, respectively. There were no approach-related complications. Clinical failures occurred in patients with bony stenosis in the lateral recess and entry zone of the neuroforamen. Less favorable outcomes were observed in patients with concomitant contained disc herniations when compared to extruded disc herniations $(P<0.03)$ as well in patients older than 50 years of age $(P<0.021)$.

\section{Conclusions}

Percutaneous, endoscopic decompression using outside-in technique works well in patients with monoradiculopathy due to lateral stenosis in the mid and exit zone of the neuroforamen. Decompression in the entry zone maybe inadequate using the transforaminal outside-in approach. Future studies with greater statistical power should determine as to whether pain relief was achieved via microdiscectomy or foraminoplasty.

keywords:

Preoperative Classification of Lumbar Foraminal Spinal Stenosis, Percutaneous endoscopic transforaminal lumbar foraminotomy, outside-in technique, microdiscectomy

Volume 8 Article 26 - Endoscopic \& Percutaneous Special Issue doi: 10.14444/1026

\section{Introduction}

Minimally invasive techniques for the treatment of lumbar spinal stenosis have found their way into mainstream spinal surgery. Many surgeons report faster recovery, rehabilitation, and return to work with the use of mini-open exposures to the lumbar spine. Endoscopic techniques are evolving and there are a number of innovative systems available whose improved optical equipment and surgical instrumentation allows for true minimally invasive percutaneous visualized foraminal decompression. ${ }^{1-4}$

This approach appears attractive for a number of reasons. First, the incision is extremely small and the surgery can be performed under local anesthesia with sedation in an outpatient setting. ${ }^{5-8}$ Second, patient expectations nowadays are high, since percutaneous procedures are the standard of care in other surgical subspecialities. Examples include the laparoscopic cholecystectomy, appendectomy, and a multitude of other general and urological procedures. Third, long-term side effects of open spinal surgery for laminectomy and/or fusion such as postlaminectomy instability and epidural fibrosis often prompts more spinal surgery later in the patient's life which further complicates the reconstructive problem both for the patient and the surgeon. Last but not least, health 
policy makers, review boards, granting agencies, and payers continue to look for services that can be provided to their beneficiaries in a more cost effective manner in an outpatient surgical facility.

While the question remains whether these perceived advantages are associated with improved clinical outcomes, it seems intuitively clear that a percutaneous, transforaminal, endoscopic approach to the compressed neural elements is by far less disturbing to the spinal motion segment than a traditional open laminectomy approach, where postlaminectomy instability and epidural fibrosis rates have been reported as high as $25 \%{ }^{9-12}$

In this study, we evaluated the clinical results with the transforaminal endoscopic decompression procedure in lumbar monoradiculopathy patients due to lateral stenosis with or without herniated disc by stratifying patients based on radiographic classification systems (CT and MRI). The surgical technique employed by the author essentially is a variation of the Thessys technique popularized by Hoogland and Schubert et al., ${ }^{7,8}$ which employs a foraminoplasty in patients with or without lateral stenosis for the treatment of herniated disc. Foraminoplasty instruments such as reamers are deployed inside the working cannula to lessen the risk of dysethesia and irritation of the exiting nerve root and its dorsal root ganglion.

\section{Materials and Methods}

\section{Patient population}

All patients provided informed consent to be included in this case series. This retrospective study included 220 consecutive patients undergoing percutaneous endoscopic foraminotomy and microdiscectomy at 228 levels. All patients were treated by a single surgeon (KUL) between 2007 and 2010. The mean follow up was 46 months ranging from 26 to 54 months. The inclusion criteria were as follows: (1) clinical signs of lumbar monoradiculopathy, dysethesias, and decreased motor function; (2) imaging evidence of foraminal or lateral recess stenosis (criteria described below) demonstrated on preoperative magnetic resonance images (MRI) and computed tomography (CT) scans; (3) unsuccessful non-operative treatment including physical therapy, and transforaminal epidural steroid injections for at least 12 weeks; (4) an age of 35-85 years. Patients were excluded from the study if they showed segmental instability on preoperative extension flexion radiographs, and had severe central stenosis (less than $100 \mathrm{~mm}^{2}$ ) (see Table 1).

Table 1. Patient population and type of lesion.

\begin{tabular}{|l|l|}
\hline \multicolumn{2}{|l|}{220 Study Patients } \\
\hline Extruded Disc & 24 Patients \\
\hline Contained Disc & 82 Patients \\
\hline Disc Bulge & 33 Patients \\
\hline Foraminal Stenosis & 114 Patients \\
\hline
\end{tabular}




\section{Preoperative Work up \& Clinical Follow up}

Preoperatively, radiographs, MR and CT images were obtained in all patients.

Postoperatively, CT images were taken if the patient showed no improvement of clinical symptoms after at least 6 weeks after surgery.

Patients returned for clinical follow-up at 6 weeks postoperatively, and at 3, 6, 12, and 24 months, respectively. At 24 months, the clinical follow up was $96 \%$. After the two-year follow up appointment, patients were seen on an annual or biannual basis. The long-term follow up after two years was less reliable and available in $67 \%$ of patients. Therefore, results reported herein were computed from data obtained at 2 year follow up. Clinical outcomes were assessed by the patient using the visual analog score (VAS) for leg pain ranging from no pain (0) to worst pain (10), and by the treating surgeon (KUL) using the Macnab criteria. ${ }^{13}$ Briefly, at latest follow up results were classified as Excellent if the patient had no pain and no limitation of activities. Outcomes were classified as Good if the patient reported occasional pain or dysethesias without any restrictions of daily activities, and did not need any pain medication. Patients were assigned to one of the two remaining categories if their pain improved somewhat but they continued to need pain medication (Fair), or if their function worsened or they needed additional surgery to address their symptoms (Poor).

\section{Radiologic Classification of Foraminal Stenosis}

Lee's classification of foraminal and lateral recess stenosis was used to define the location of the offending pathology within the neuroforamen by dividing it from medial to lateral into entry (dura to pedicle; zone 1), middle (medial pedicle wall to center pedicle; zone 2), and exit zone (center pedicle to lateral border of the facet joint; zone 3 ). ${ }^{14}$ Foraminal and lateral recess stenosis were stratified according to the main offending pathology: extruded herniated disc, disc bulge, and bony stenosis. In the entry zone, Lee described hypertrophy of the superior articular face as the predominant pathology. In the mid-zone, it was often due to an osteophytic process underneath the pars interarticularis, and in the exit zone due a subluxed and hypertrophic facet joint (Figure 1). ${ }^{14}$ 


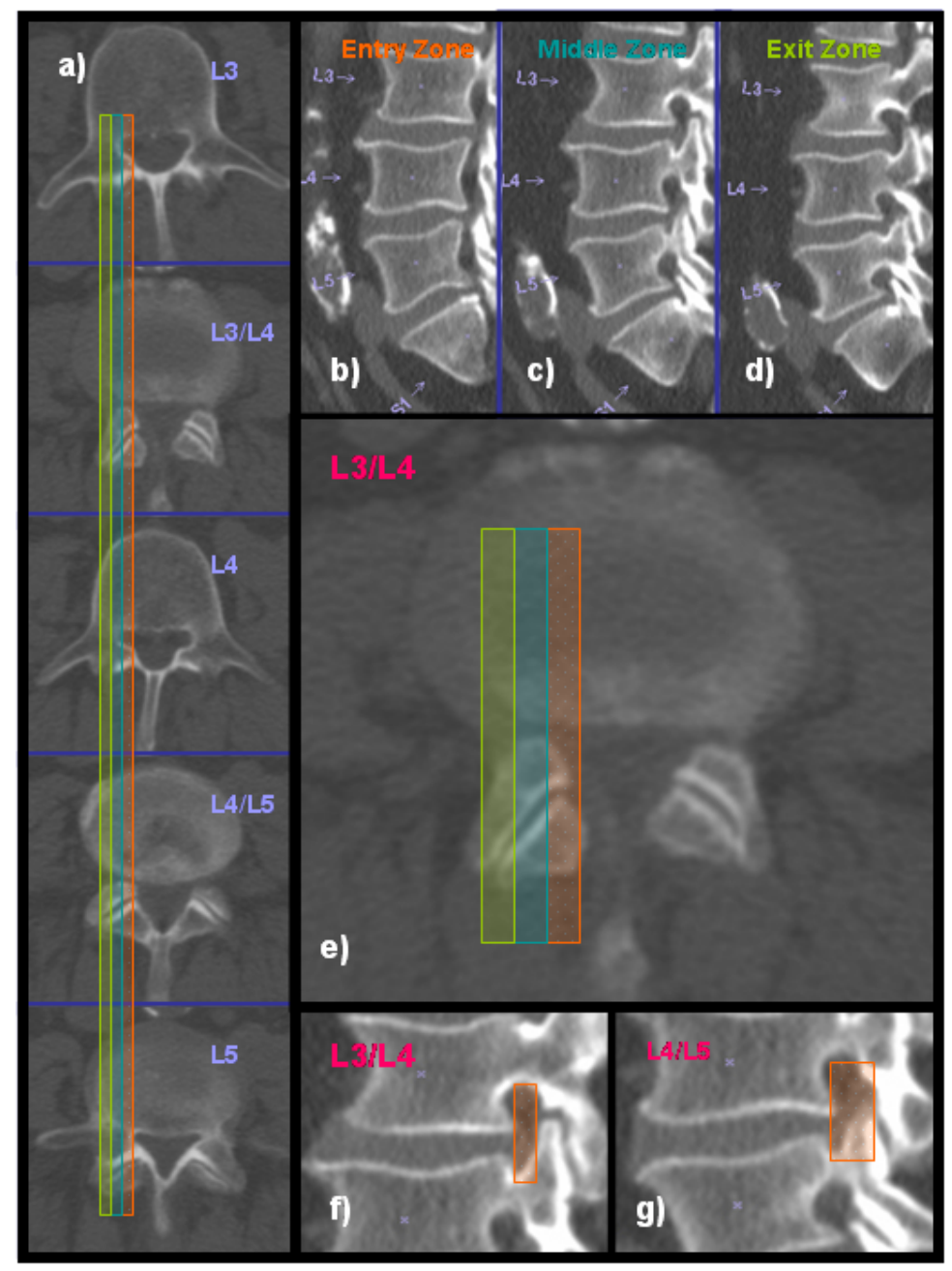

Fig. 1. Preoperative CT scans of a 70 year old male: a) panel on the left shows axial CT cuts from L3 to L5, b-d) panel shows sagittal CT cuts through the entry (shaded orange), middle (shaded turquoise), and exit zone (shaded green) of the lumbar neuroforamina, e) axial CT cut through the L3-4 disc space showing the stenotic lesion in the middle zone at that level, $\mathrm{f}-\mathrm{g}$ ) sagittal CT cuts through the middle zone at L3-4, and the L4-5 level. The neuroforaminal height (orange shade area) is less than $5 \mathrm{~mm}$. The neuroforaminal width is less than $2 \mathrm{~mm}$. Both indicators are consistent with spinal stenosis.

The height of the posterior intervertebral disc and lumbar foramina was evaluated according to Hasegawa, ${ }^{15}$ who described a lumbar neuroforaminal height of $15 \mathrm{~mm}$ or more as normal, reduced posterior intervertebral disc height of 3 to $4 \mathrm{~mm}$ as suggestive of spinal stenosis. Preoperative sagittal and axial MRI and CT images were used to assess the location and extent of foraminal stenosis. Only patients with stenotic lesions (regardless whether due to bony stenosis or extruded disc herniation or contained disc bulge) producing a neuroforaminal width of $3 \mathrm{~mm}$ or less on the sagittal MRI and CT cuts or lateral recess height of $3 \mathrm{~mm}$ or less on the axial MRI and CT cuts were included in this analysis. 
Only one predominant zone of foraminal stenosis was assigned per patient to stratify analysis (Figure 2). Disc herniations involving the lateral recess were grouped as either extruded, or contained.

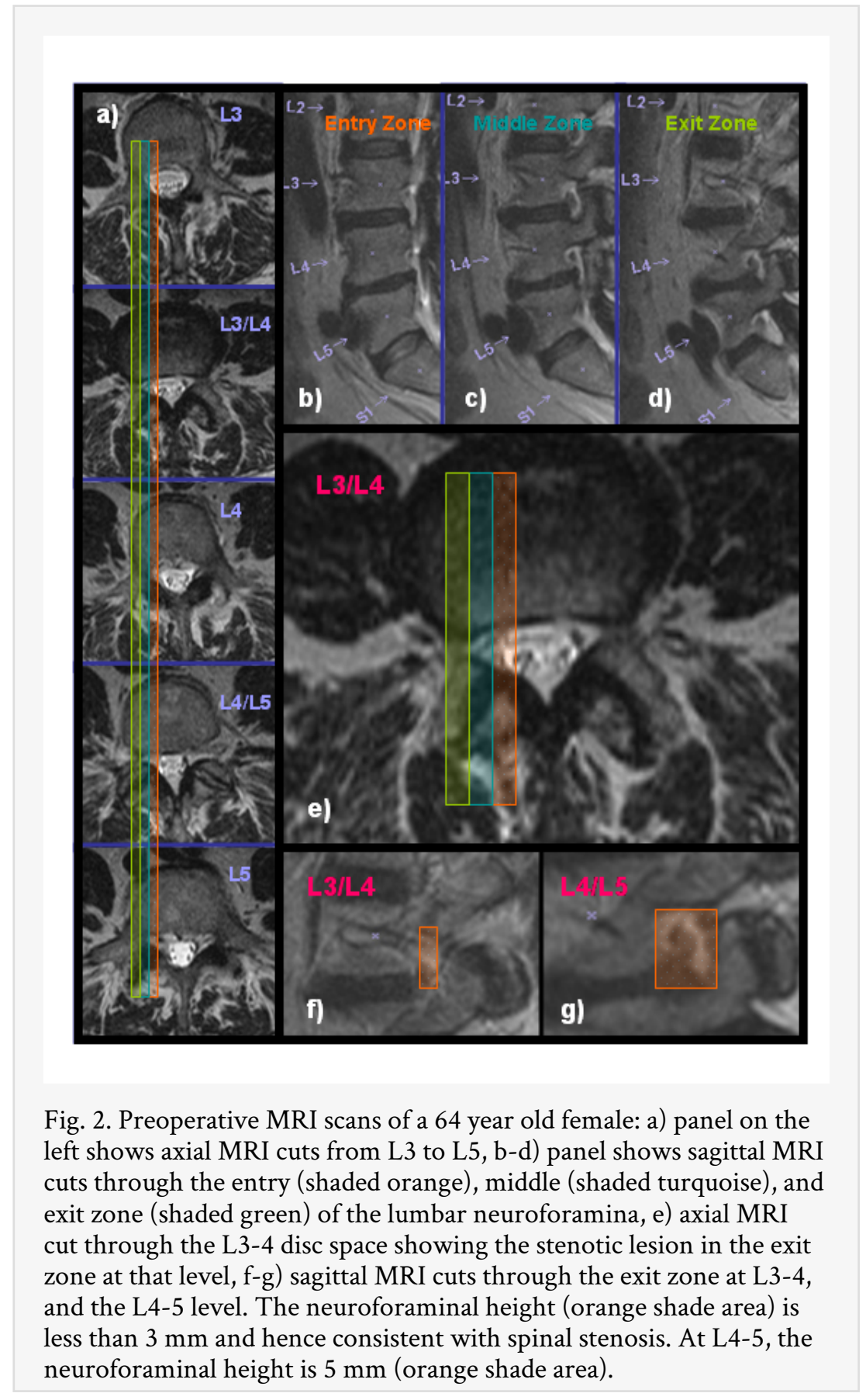

\section{Surgical Techniques}

All surgical procedures were performed with the endoscopic transforaminal approach using the "outside-in" technique, in which the working sheath is placed into the lower portion of the neuroforamen, thus, retracting and avoiding the exiting nerve root. No part of the cannula tip or the endoscope is positioned in the disc space. The surgical technique employed by the author essentially is a variation of the Thessys technique popularized by 
Hoogland and Schubert et al., ${ }^{7,8}$ which employs a foraminoplasty in patients with or without lateral stenosis for the treatment of herniated disc. For the foraminoplasty, reamers and drills are deployed inside the working cannula to lessen the risk of dysethesia and irritation of the exiting nerve root and its dorsal root ganglion.

Procedures were performed in prone position under local anesthesia and sedation in all patients. In some instances, where access to the L5-S1 neuroforamen was difficult due to a high riding ilium, patients were positioned in the lateral decubitus position. Techniques to define the skin entry point and the surgical trajectory have been described elsewhere. ${ }^{1,4}$ Entry points were generally laterally at 7-9 $\mathrm{cm}$ at the L3-4 level, $8-10 \mathrm{~cm}$ at the L4-5 level, and $10-12 \mathrm{~cm}$ at the L5-S1 level.

The targeted neuroforamen was accessed as follows: First, an 18-G (150 mm in length) needle is inserted into the safe zone of Kambin's triangle bordered by the traversing nerve root medially, the exiting nerve root laterally, and the lower adjacent pedicle distally. 5 Ideally, the targeting needle was placed on the lateral view into the lower portion of the neuroforamen into the disc. On the anterior-posterior view, the needle tip should be at the medial interpedicular line. A steel guide wire was then inserted into the intervertebral disc and the $18-\mathrm{G}$ spinal needle was removed. Dilators, drills and trephines of increasing diameters were used for foraminal decompression procedures. Additional cannulated reamers measuring 7 and $9 \mathrm{~mm}$ in diameter intended to be used over a guide wire without the protective working cannula were available but rarely to further minimize risk of dysesthesia of the exiting nerve root and irritation of its dorsal root ganglion (see Table 2).

Table 2. Foraminoplasty instruments.

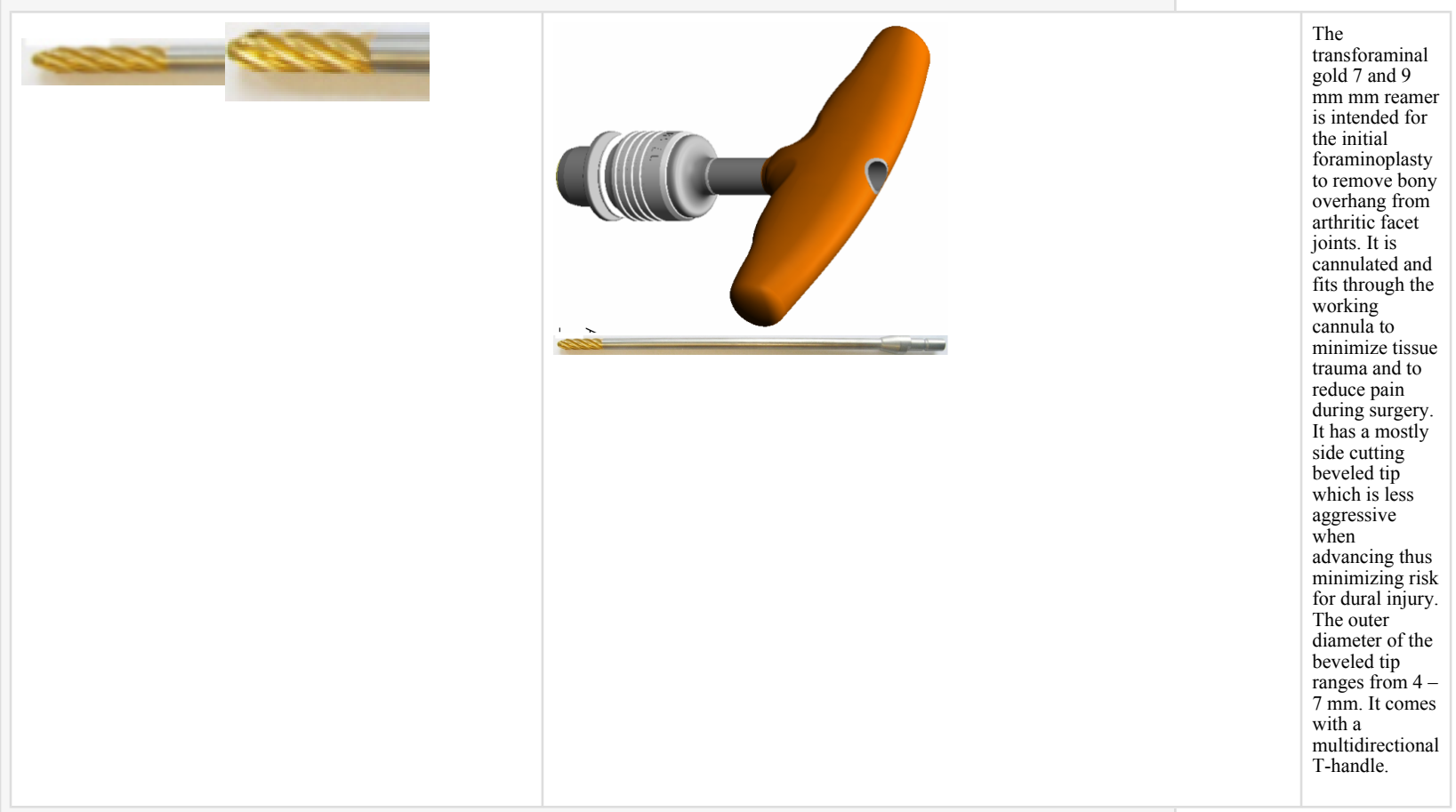




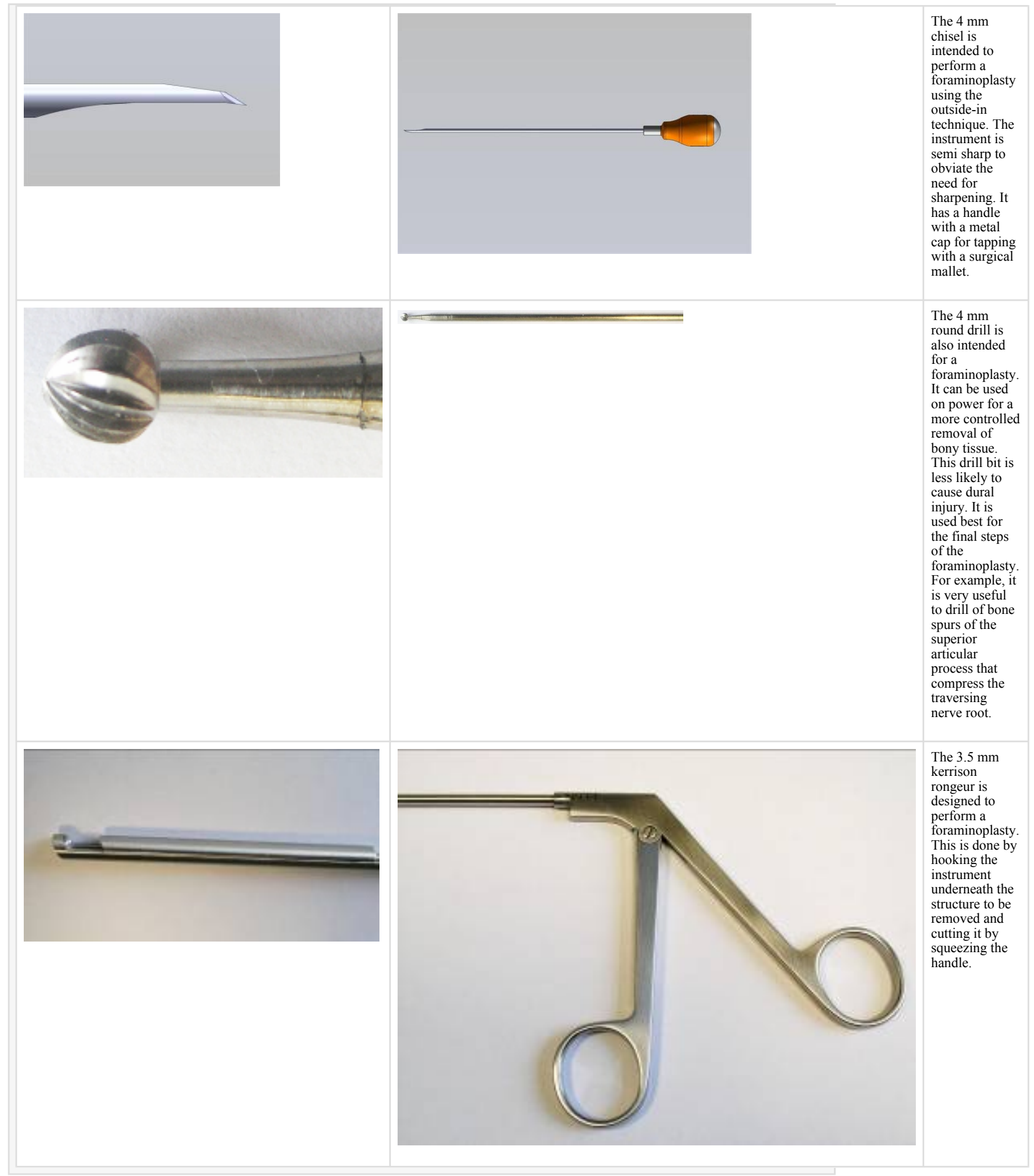




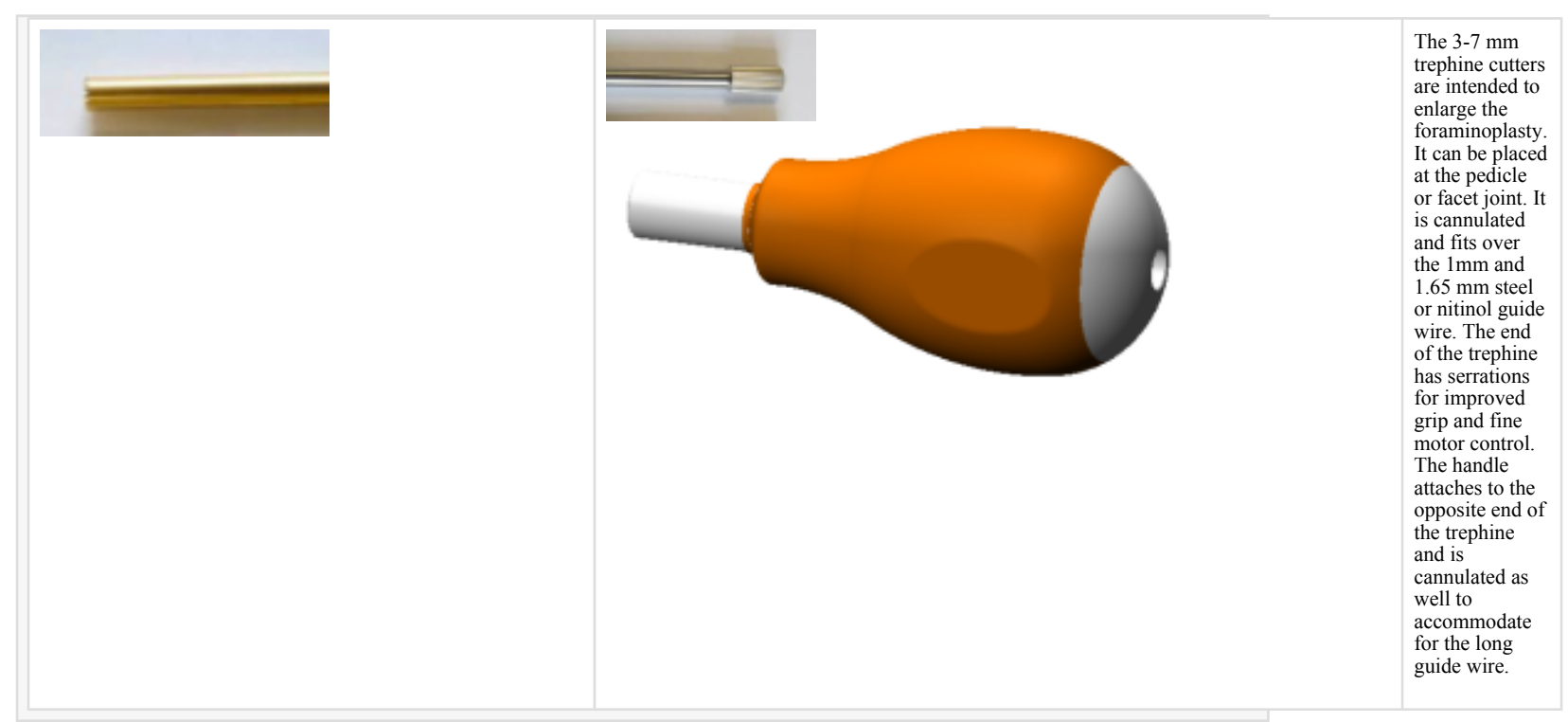

For the foraminoplasty, bone from the hypertrophied superior and inferior articular process was removed with different instruments including endoscopic chisels, drills, Kerrison rongeurs, and percutaneous trephines (Figure 3, Figure 4, Figure 5, Figure 6). The foraminoplasty was facilitated by changing the trajectory of the instruments to aim for the compressive pathology identified on preoperative studies. In case of concomitant herniated disc, extruded disc material was removed using forceps and pituitary rongeurs and contained herniations were decompressed through a small annular window. Epidural bleeding was controlled with a radiofrequency probe (Ellman; Ellman International LLC, USA) under saline irrigation. 


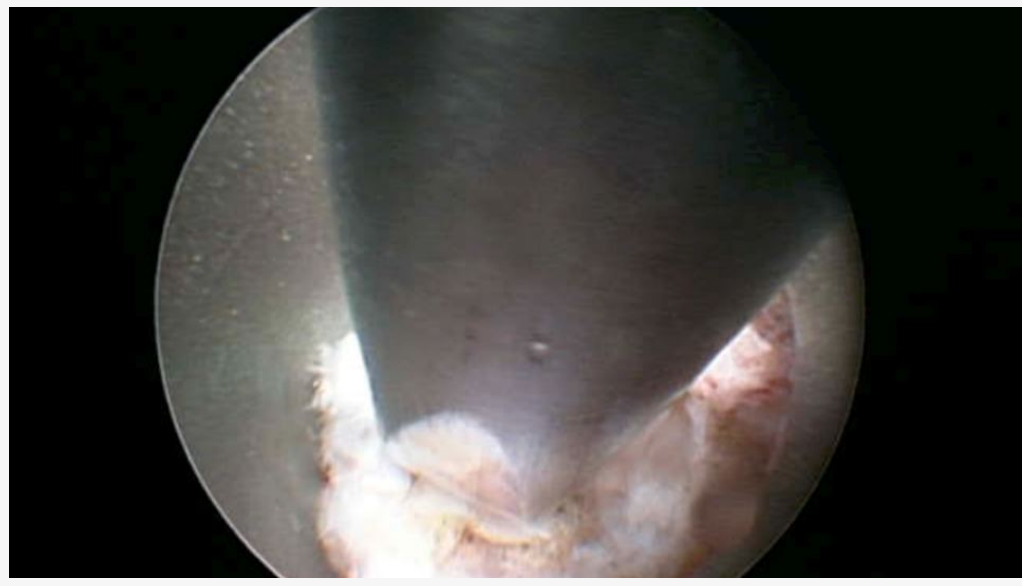

Fig. 3. Endoscopic view of the chisel used to perform a foraminoplasty. The cannula is docked at the lateral superior aspect of the facet joint. The chisel is introduced through the working channel of the endoscope starting in an upward direction and then chiseling in a downward direction by rotating the chisel 180 degrees to remove bone from the facet joint.

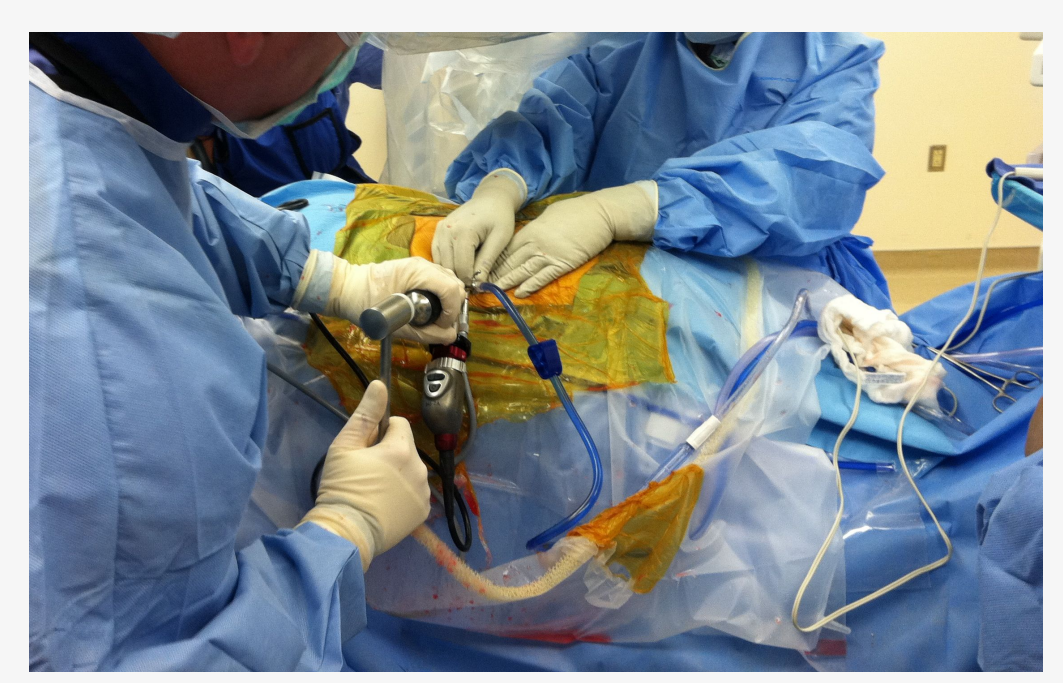

Fig. 4. The chisel is advanced through the central working channel of the endoscope. A mallet may be used to advance the chisel for the foraminoplasty. Typically, a direct lateral approach to the foramen by dropping one's hand is more advantageous. The foraminoplasty can be facilitaed by chiseling in an upward diretion, then by rotating the chisel by 180 degrees followed by downward chiseling. 


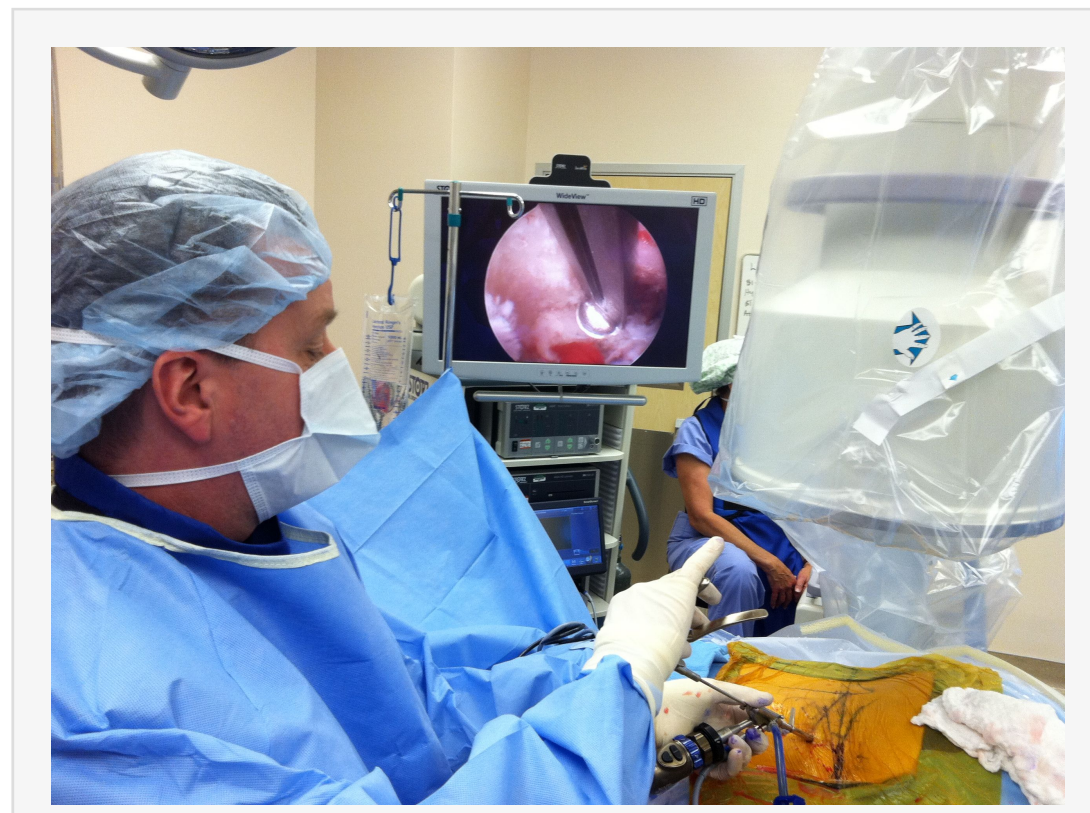

Fig. 5. An endoscopic Kerrison rongeur can be used to finalize the foraminoplasty. It is most suitable for decompressing the most medial portion of the lateral recess by using a Kerrison with a 135 degree footplate. The Kerrison is directed past the leading edge of the facet joint, then rotated 180 degrees to remove bone by dropping one's hand. 


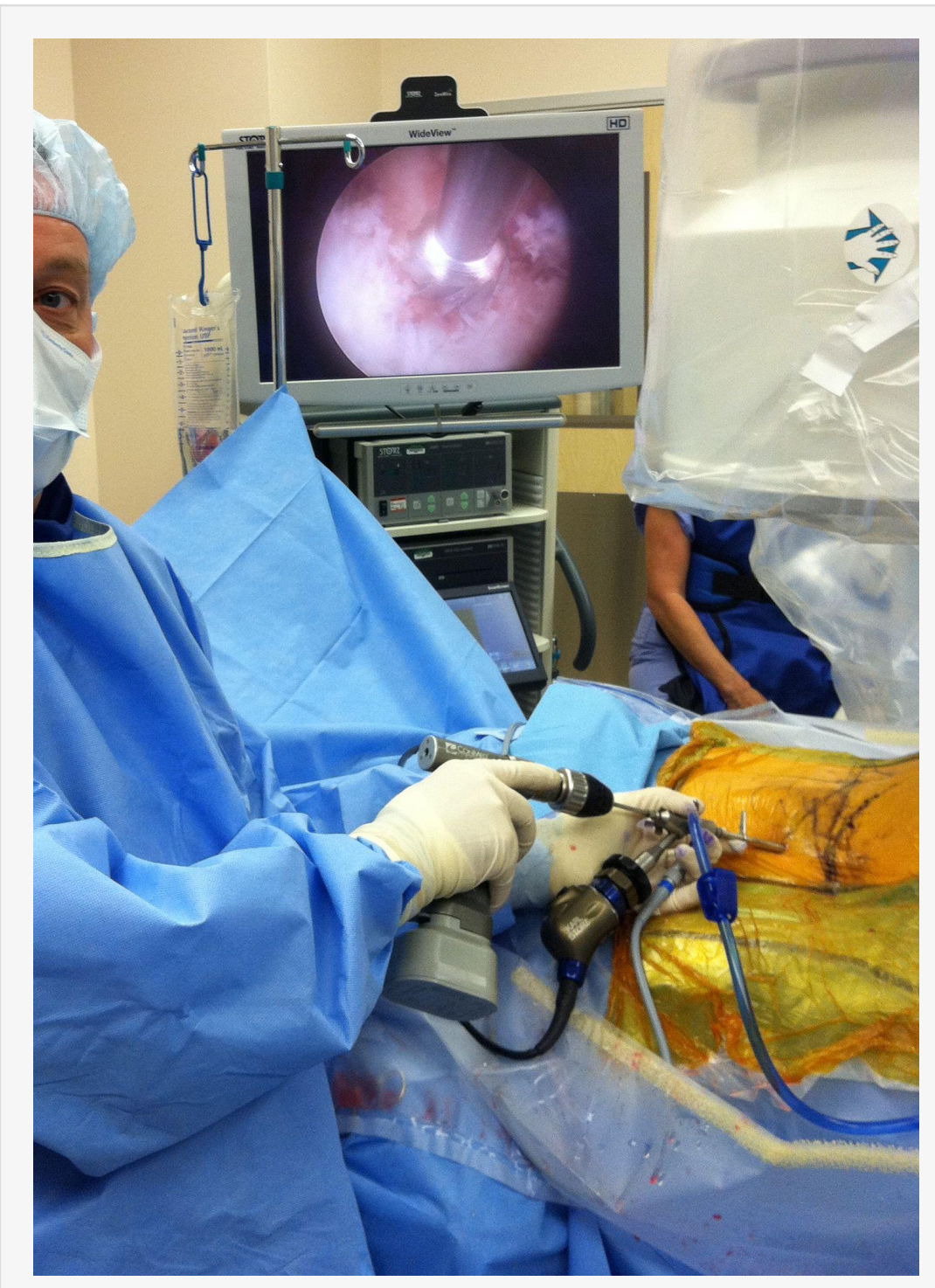

Fig. 6. A foraminal drill can be advanced directly to the inner working channel. The drill is attached to a power driver and can be used in forward and reverse. It is most suitable for expansile foraminoplasty around the inferior pedicle.

\section{Statistical Methods}

Crosstabulation statistics and measures of association were computed for two-way tables using SPSS Version 15.0. Using the modified McNab criteria and foraminal zone classification as row and column variables, and age (over 50 and under 50 years of age) as control variable (layer factor), the crosstabulation procedure was employed to form one panel of associated statistics and measures for each value of the layer factor (or a combination of values for two or more control variables). This correlation matrix allowed to calculate expected counts of variable combinations if no association was found between clinical outcome and zone classification of foraminal stenosis and variable distribution was equal. The Pearson chi-square, and the likelihood-ratio chi-square tests were used as statistical measures of association. 


\section{Results}

There were 220 patients consisting of 132 females, and 88 males. The average age was 52.4 ranging from 37 to 86 . Of the study population, 212 patients underwent a singlelevel operation and 8 patients had a two-level operation. Thus, a total of 228 levels were operated in 220 patients.

The L4-5 segment was the most commonly involved level (132 levels, $57.9 \%$ ) followed by L5-S1 (62 levels, $27.2 \%$ ), L3-4 (31 levels, $13.6 \%$ ), and the L2/3 level (3 levels, $1.3 \%)$.

According to the Macnab criteria, excellent and good results were obtained in $85 \%$ (186/ 220) of patients with monoradiculopathy. The mean VAS score decreased from $7.5 \pm 1.5$ preoperatively to $2.8 \pm 1.9$ at the final follow-up $(P<0.01)$. Concomitant extruded disc herniations were recorded in 24 patients and 82 patients had contained disc bulges. There were no approach-related complications.

In patients with concomitant extruded disc fragments (24/220), the mean VAS score decreased from $7.2 \pm 1.4$ preoperatively to $2.3 \pm 1.6$ at the final follow-up $(P<0.01)$. Patients with concomitant contained disc bulges $(82 / 220)$ had excellent and good results $60.4 \%$ of the time. In this group, the mean VAS score decreased from $7.8 \pm 1.9$

preoperatively to $3.3 \pm 1.8$ at the final follow-up $(P<0.01)$. Hence, outcomes in patients with concomitant contained disc bulges were less favorable when compared to outcomes in patients with concomitant extruded disc herniations $(P<0.03)$.

Patients treated for bony foraminal stenosis alone, $71.9 \%(114 / 220)$ had excellent to good results. Eighty-two of these 114 patients had middle and exit zone foraminal stenosis. The remaining 32 patients with fair \& poor clinical results occurred in patients with entry zone foraminal stenosis. The differences in clinical outcomes were statistically significant $(P<$ 0.005). Age over 50 years was identified as an additional risk factor for fair to poor outcomes with statistical significance $(P=0.021)$. Twenty-two of the 32 patients with foraminal stenosis in the entry zone and fair to poor clinical outcomes were over 50 years of age. There were no approach-related complications.

\section{Discussion}

Preoperative planning is essential in achieving excellent and good outcomes with the transforaminal endoscopic surgery for foraminal stenosis. Although the effectiveness of the procedure has been demonstrated by multiple investigators, ${ }^{1-12,15-18}$ clinical data are considered less favorable for bony foraminal stenosis than for herniated disc, where clinical success rates upwards of $90 \%$ are expected. Patients with unilateral monoradiculopathy due to neural element compression in the lateral recess or neuroforamen treated with transforaminal decompression utilizing the outside-in technique with foraminoplasty did well. In addition, our study suggests that bony foraminal and lateral recess stenosis can be treated successfully with the transforaminal approach if an extensive foraminoplasty is performed and patients are carefully selected. 
Recent advances in optic and endoscopic technologies allowing better visualization and more aggressive decompression of advanced, complex degenerative stenotic lesions have expanded the indications of the endoscopic transforaminal procedure. The outside-in technique lends itself favorably to treat foraminal and lateral recess stenosis as it allows direct transforaminal access to pedicle, and hypertrophied superior articular process without having to access the intervertebral disc when starting the procedure. Lee et al. have described as "epiduroscopic technique". ${ }^{18}$ Bony decompression through the working cannula using drills, chisels, reamers, and rongeurs may better protect the exiting nerve root and, hence, decrease the risk of postoperative dysethesia and irritation of its dorsal root ganglion. The author's technique is essentially a modification of the Thessys technique popularized by Hoogland and Schuber et al. ${ }^{7,8}$ The author's preferred technique is to use Kerrison rongeurs, chisels, and power drills that can be introduced directly through the central working channel of the endoscope. The decompression can be all done under direct visualization and the neural structures can be safely retracted. The original Thessys technique advocates the use of transforaminal percutaneous reamers and drills, which are introduced for non-visualized lateral facet resection over a guide wire without the use of an endoscopic visualized technique described by Yeung, et al. ${ }^{1-4}$ They are intended to be used under fluoroscopic guidance and carry the risk of injury to the exiting and traversing nerve root, which may produce dysethetic leg pain and neurological dysfunction in the affected extremity.

Since sciatica was the main reason for surgical intervention, reduction of leg pain was analyzed using a VAS. There was a significant improvement in the VAS and the clinical outcome at final follow up. Our clinical outcomes are comparable to success rates reported by patients undergoing laminectomy for spinal stenosis. ${ }^{19,20}$ The presence of a concomitant extruded disc herniation or a contained disc bulge was recorded. However, this study lacked sufficient statistical power to analyze whether postoperative pain relief was produced by the bony decompression or the potential rhizotomy effect of the lateral facet resection during the foraminoplasty or the microdiscectomy. The latter would presumably treat a discogenic pain component. Future studies with more statistical power should investigate this further.

The importance of preoperative planning of transforaminal endoscopic removal of herniated discs, has been stressed by Lee et al. who suggested a classification based on the location of a migrated disc fragment. ${ }^{18}$ In this study, we employed a previously published radiographic classification systems ${ }^{14,15}$ in the preoperative decision making in patients with symptomatic lateral stenosis and correlated them with clinical outcomes according to the modified McNab criteria. ${ }^{13}$ In 1988, Lee published on a three zone classification of the neuroforamen by dividing it into entry, middle, and exit zone. ${ }^{14}$ Moreover, the height of the neuroforamen underneath the articular processes of the lumbar facet joint can be used to classify to spinal stenosis. In 1995, Hasegawa defined the height of the posterior intervertebral disc height of $5 \mathrm{~mm}$ or more as normal. ${ }^{15} \mathrm{He}$ suggested that a reduced height of 3 to $4 \mathrm{~mm}$ is suggestive of spinal stenosis. He found that a posterior intervertebral disc height of $2 \mathrm{~mm}$ or less and neuroforaminal height of less than $15 \mathrm{~mm}$ is associated with nerve root compression approximately $80 \%$ of the time. 
As demonstrated by this study, application of radiographic grading systems of foraminal stenosis may assist in selecting appropriate surgical candidates for the transforaminal decompression procedure. Our results indicated that patients with stenosis in the entry zone of the neuroforamen did worse than those with stenosis in the middle and exit zone. These types of stenotic lesions should perhaps be treated with laminectomy instead. Our clinical series clearly demonstrates the successful use of reamers, chisels, drills, and trephines that may be positioned under direct visualization through the center working channel of the endoscope and, thus, may allow a more sophisticated endoscopic decompression.

\section{Conclusion}

Percutaneous endoscopic transforaminal decompression for monoradiculopathy employing the outside-in technique with expansile foraminoplasty is feasible and works well in patients with lateral recess stenosis in the mid and exit zone of the neuroforamen. Decompressive surgery through a laminectomy approach should be considered for neuroforaminal stenosis in the entry zone. Regardless of the instrumentation, preoperative classification of the neuroforamen into three zones may prove useful in the preoperative patient selection process.

\section{References}

1. Yeung AT, Yeung CA. Minimally invasive techniques for the management of lumbar disc herniation. Orthop Clin North Am. 2007 Jul;38(3):363-72.

2. Tsou PM, Alan Yeung C, Yeung AT. Posterolateral transforaminal selective endoscopic discectomy and thermal annuloplasty for chronic lumbar discogenic pain: a minimal access visualized intradiscal surgical procedure. Spine J. 2004 SepOct;4(5):564-73.

3. Tsou PM, Yeung AT. Transforaminal endoscopic decompression for radiculopathy secondary to intracanal noncontained lumbar disc herniations: outcome and technique. Spine J. 2002 Jan-Feb;2(1):41-8.

4. Yeung AT, Yeung CA. Advances in endoscopic disc and spine surgery: foraminal approach. Surg Technol Int. 2003;11:255-63.

5. Kambin P, Casey K, O’Brien E, et al (1996) Transforaminal arthroscopic decompression of lateral recess stenosis. J Neurosurg 84:462-467.

6. Kambin P, O'Brien E, Zhou L (1998) Arthroscopic microdiscectomy and selective fragmentectomy. Clin Orthop 347:150-167

7. Hoogland T, Schubert M, Miklitz B, Ramirez A. Transforaminal posterolateral endoscopic discectomy with or without the combination of a low-dose chymopapain: a prospective randomized study in 280 consecutive cases. Spine. 2006 Nov 15;31(24):E890-7.

8. Schubert M, Hoogland T. Endoscopic transforaminal nucleotomy with foraminoplasty for lumbar disk herniation. Oper Orthop Traumatol. 2005 Dec;17(6):641-61.

9. Schaller B. Failed back surgery syndrome: the role of symptomatic segmental singlelevel instability after lumbar microdiscectomy. Eur Spine J. 2004 May;13(3):193-8. 
10. Mullin BB, Rea GL, Irsik R, Catton M, Miner ME. The effect of postlaminectomy spinal instability on the outcome of lumbar spinal stenosis patients. J Spinal Disord. 1996 Apr;9(2):107-16.

11. Papagelopoulos PJ, Peterson HA, Ebersold MJ, Emmanuel PR, Choudhury SN, Quast LM. Spinal column deformity and instability after lumbar or thoracolumbar laminectomy for intraspinal tumors in children and young adults. Spine. $1997 \mathrm{Feb}$ 15;22(4):442-51.

12. Mullin BB, Rea GL, Irsik R, Catton M, Miner ME. The effect of postlaminectomy spinal instability on the outcome of lumbar spinal stenosis patients. J Spinal Disord. 1996 Apr;9(2):107-16.

13. Macnab I (1971) Negative disc exploration An analysis of the causes of nerve-root involvement in sixty-eight patients. J Bone Joint Surg Am 53:891-903.

14. Lee CK, Rauschning W, Glenn W. Lateral lumbar spinal canal stenosis: classification, pathologic anatomy and surgical decompression. Spine. 1988 Mar;13(3):313-20.

15. Hasegawa T, An HS, Haughton VM, Nowicki BH. Lumbar foraminal stenosis: critical heights of the intervertebral discs and foramina. A cryomicrotome study in cadavera. J Bone Joint Surg Am. 1995 Jan;77(1):32-8.

16. Kim MJ, Lee SH, Jung ES, Son BG, Choi ES, Shin JH, Sung JK, Chi YC. Targeted percutaneous transforaminal endoscopic diskectomy in 295 patients: comparison with results of microscopic diskectomy. Surg Neurol. 2007 Dec;68(6):623-31.

17. Ahn Y, Lee SH, Park WM, Lee HY, Shin SW, Kang HY. Percutaneous endoscopic lumbar discectomy for recurrent disc herniation: surgical technique, outcome, and prognostic factors of 43 consecutive cases. Spine. 2004 Aug 15;29(16):E326-32.

18. Lee S, Kim SK, Lee SH, Kim WJ, Choi WC, Choi G, Shin SW. Percutaneous endoscopic lumbar discectomy for migrated disc herniation: Classification of disc migration and surgical approaches. Eur Spine J. 2007 Mar;16(3):431-7.

19. Fokter SK, Yerby SA. Patient-based outcomes for the operative treatment of degenerative lumbar spinal stenosis. Eur Spine J. 2006 Nov;15(11):1661-9.

20. Sengupta DK, Herkowitz HN. Lumbar spinal stenosis. Treatment strategies and indications for surgery. Orthop Clin North Am. 2003 Apr;34(2):281-95.

\section{Corresponding Author}

Kai-Uwe Lewandrowski, MD, Center for Advanced Spinal Surgery of Southern Arizona, P.O. Box 64217, Tucson, AZ 85728-4217. kaiuwe19@gmail.com

\section{Disclosures}

Dr. Lewandrowski consults for Amendia and Integra Life Science.

Copyright (C) 2014 ISASS - International Society for the Advancement of Spine Surgery. To see more or order reprints or permissions, see http://ijssurgery.com. 